\title{
Maria LOS
}

criminologue, Département de criminologie,

Université d’Ottawa

(1983)

\section{"Les femmes, le pouvoir et le crime en Pologne"}

Un document produit en version numérique par Jean-Marie Tremblay, bénévole, professeur de sociologie au Cégep de Chicoutimi

Courriel: jean-marie_tremblay@uqac.ca

Site web pédagogique : http://www.uqac.ca/jmt-sociologue/

Dans le cadre de la collection: "Les classiques des sciences sociales"

Site web: http://classiques.uqac.ca/

Une collection développée en collaboration avec la Bibliothèque

Paul-Émile-Boulet de l'Université du Québec à Chicoutimi

Site web: http://bibliotheque.uqac.ca/ 
Cette édition électronique a été réalisée par Jean-Marie Tremblay, bénévole, professeur de sociologie au Cégep de Chicoutimi à partir de :

\section{Maria LOS}

criminologue, Centre international de criminologie comparée, Université de Montréal

“Les femmes, le pouvoir et le crime en Pologne”.

Un article publié dans la revue Criminologie, vol. 16, no 2, 1983, pp. 47-66. Montréal: Les Presses de l'Université de Montréal. Numéro intitulé : Les femmes et la justice pénale.

[Autorisation formelle accordée par Mme Maria LOS, criminologue, de diffuser cet article dans Les Classiques des sciences sociales, le 29 mars 2007.]

gy Courriel : marialos@uOttawa.ca

Polices de caractères utilisée :

Pour le texte: Times New Roman, 14 points.

Pour les citations : Times New Roman, 12 points.

Pour les notes de bas de page : Times New Roman, 12 points.

Édition électronique réalisée avec le traitement de textes Microsoft Word 2004 pour Macintosh.

Mise en page sur papier format : LETTRE (US letter), 8.5'’ x 11'’)

Édition numérique réalisée le 23 mai 2007 à Chicoutimi, Ville de Saguenay, province de Québec, Canada.

\section{Fait avec}




\section{Maria LOS}

criminologue, Centre international de criminologie comparée,

Université de Montréal

\section{"Les femmes, le pouvoir et le crime en Pologne”}

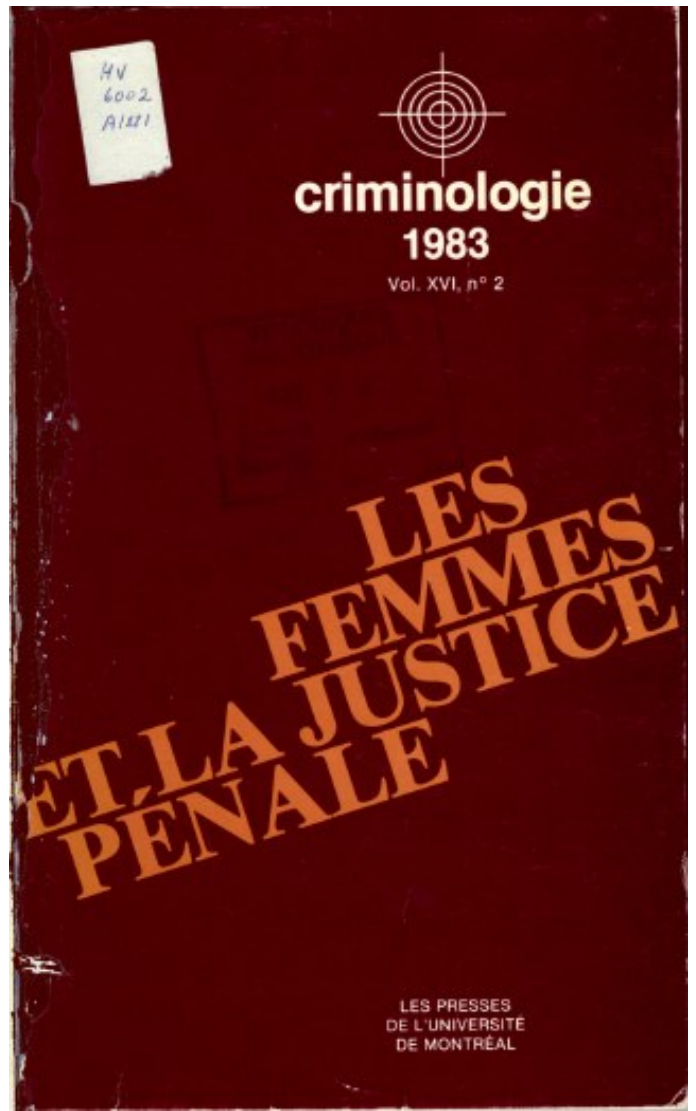

Un article publié dans la revue Criminologie, vol. 16, no 2, 1983, pp. 47-66. Montréal: Les Presses de l'Université de Montréal. Numéro intitulé : Les femmes et la justice pénale. 


\section{Table des matières}

$\underline{\text { Introduction }}$

1. Les contributions récentes

2. L'image statistique de la criminalité féminine

3. L'émancipation de la femme

4. L'émancipation de la femme et le crime : les années d'après-guerre

5. L'émancipation de la femme et le crime : les vingt dernières années

6. L'émancipation de la femme : discussion

Bibliographie 
Maria LOS *

\section{"Les femmes, le pouvoir et le crime en Pologne”}

Un article publié dans la revue Criminologie, vol. 16, no 2, 1983, pp. 47-66. Montréal: Les Presses de l'Université de Montréal. Numéro intitulé : Les femmes et la justice pénale.

(traduction libre de l'anglais)

\section{Introduction}

\section{$\underline{\text { Retour à la table des matières }}$}

En Pologne, comme ailleurs, les hommes semblent plus attirés par le crime que les femmes, ou, tout au moins, s'arrangent-ils pour figurer officiellement plus souvent dans les statistiques.

Un nombre de plus en plus grand d'ouvrages criminologiques s'intéressent à l'importance du sexe dans la criminalité et le système de justice pénale. Cela implique généralement l'analyse des différences culturelles et structurelles dans les rôles sexuels aussi bien que les pratiques sexistes des agences de justice pénale œuvrant dans une société construite d'après les intérêts des hommes (Smart, 1977a, b; Hagan et al., 1979 ; Bertrand, 1979). La Pologne offre un cas intéressant aux étudiants de la criminalité des femmes, car la femme et l'homme polonais ont tous deux des niveaux d'instruction et une implication à l'ex-

* Professeure de criminologie à l'Université d'Ottawa. 
térieur de la maison très semblables. De plus, le système de justice pénale emploie un très grand nombre de femmes.

Ceci dit, la situation polonaise informera-t-elle les théories prédisant l'augmentation significative de la délinquance des femmes en fonction du processus de libération de la femme et de son intégration au sein des structures officielles et organisées de la société ? Des théories superficielles peuvent être facilement corroborées ou contredites par une généralisation superficielle des faits.

De tels exercices, cependant, ne contribuent pas tellement àla compréhension d'une réalité sociale complexe. Deux articles (Adler, 1977 ; Plenska, 1980) qui tentent d'expliquer pourquoi l'émancipation de la femme en Pologne n'a pas provoqué un accroissement significatif de la criminalité féminine, touchent à certains aspects et facteurs importants reliés à cette question niais en négligent d'autres. Le présent article constitue une contribution à la discussion qu'ils ont déjà engagée. Nous tenterons d'aborder le problème sous l'angle phénoménologique, en reconstruisant quelques-uns des aspects subjectifs des changements objectifs de l'organisation sociale. Il ne s'agit donc ni d'une rigoureuse analyse empirique ni d'une synthèse des études criminologiques dans le domaine puisque ni l'une ni les autres n'ont jamais été entreprises ${ }^{1}$. Nous nous référons aux statistiques officielles (Plenska, 1980, 468-470) comme à un point de départ nécessaire pour discuter des changements dans l'étendue et la nature des condamnations féminines.

1 Avant d'écrire cet article, j'ai parcouru chaque numéro de la revue féminine kobieta i Zycie (femme et vie) parue entre avril 81 et janvier 83. La criminalité féminine n'était pas mentionnée une seule fois. Seul un court article s'intéressait à l'alcoolisme chez la femme et un rapport traitait en termes très idéalistes la situation dans un centre de détention pour filles délinquantes. Cependant il $\mathrm{y}$ avait plusieurs articles qui présentaient les femmes comme victimes des hommes, soit qu'elles subissent une exploitation économique, qu'elles soient battues ou abandonnées comme épouses d'alcooliques ou tourmentées et accablées par leurs fils drogués ou alcooliques. Les problèmes du crime économique, de la prostitution, des abus de drogues ou d'alcool ou de toute autre forme de délinquance commise par les femme,,, n'ont jamais été jugés dignes d'attention. 


\section{LES CONTRIBUTIONS RÉCENTES}

\section{$\underline{\text { Retour à la table des matières }}$}

Alder et Plenska ont noté toutes deux que le rapport entre le taux de criminalité des femmes et celui des hommes en Pologne a augmenté quelque peu au cours de la période d'après guerre, quand les femmes s'intégrèrent au courant socio-économique, mais qu'il a diminué après quelque 10 ans. Alder croit que les contrôles normatifs exercés sur les femmes par l'Église et l'État ont continué d'opérer et que l'émancipation de la femme ne les a affectés que temporairement. En d'autre termes, la promotion professionnelle n'a pas, en fait, été suivie d'une libération psychologique et morale de la femme.

Plenska semble partager ces vues. Elle va même plus loin, affirmant que la hausse du nombre des condamnations féminines dans la période d'industrialisation intense de l'après-guerre est due à l'augmentation des occasions de commettre des crimes contre la propriété de l'État. Selon les statistiques, les crimes économiques contribuent considérablement à l'accroissement de la délinquance féminine en Pologne. Plenska attribue la dimension subséquente du taux de condamnation des femmes, partiellement, aux changements législatifs de 1953 qui ont décriminalisé les délits mineurs contre la propriété de l'État. Elle note aussi que, tandis que le droit pénal polonais a été traditionnellement orienté vers le contrôle de la déviance masculine, la prostitution et autres « délinquances morales » n'ont pas été criminalisées, et donc les augmentations de ces types de déviance ont pu passer inaperçues. 


\section{L'IMAGE STATISTIQUE DE LA CRIMINALITÉ FÉMININE}

$\underline{\text { Retour à la table des matières }}$

Selon les statistiques officielles, en 1977, il y avait 11 condamnations de femmes pour 102 d'hommes par 10000 habitants, soit un rapport de $1: 9$. Ce rapport se maintenait au cours des années 1970, mais cependant il était de $1: 5$ à $1: 8$ dans les années 60 . Et de $1: 3$ à $1: 40$ dans les années 50. En 1977, 10,2\% (14 059 cas) ${ }^{2}$ de toutes les condamnations concernaient des femmes. Comme ces condamnations comptaient pour 4,6\% (680 cas) des condamnations totales pour crimes contre la vie et la santé, 5,6\% (136 cas) pour crimes contre la liberté et seulement 2,6\% (411 cas) pour crimes contre la famille et la jeunesse, la part de la femme dans les condamnations pour crime économique était de 58,4\% (2 414 cas) et 30,7\% (536 cas) pour délit fiscal. Les condamnations féminines pour crimes contre les propriétés privées et de l'État constituent 11,1\% (4 487 cas), alors que les crimes contre les institutions et l'État constituent 11,1\% (748 cas) de toutes les condamnations pour ce crime.

Pour comprendre la signification légale de ces données, on doit étudier les types particuliers de délits où les femmes semblent être plus nombreuses. En dépit de la faible représentativité générale des femmes condamnées pour des crimes contre la propriété publique, leur participation augmente rapidement quand on exclut les crimes qui comportent l'usage de la force ou de la violence (les condamnations des femmes pour cette catégorie de délits ne comptent que pour 1,8\% de toutes les condamnations relatives à ces crimes). Ainsi, les condamnations des femmes constituent jusqu'à 32,3\% (1970 cas) de toutes les condamnations en 1977 pour usage illégal du bien public qu'elles avaient mission d'administrer, de protéger ou de gérer (ce crime est passible d'emprisonnement d'une durée de 1 à 10 ans). De

2 La Pologne a une population d'environ 35 millions. 
plus, on doit remarquer que les femmes comptent pour 20,2\% (82 cas) de toutes les condamnations pour usurpation de la propriété sociale de valeur considérable. Il s'agit d'un crime très sérieux et qui entraîne une peine de 5 à 25 ans d'emprisonnement. De plus, les condamnations des femmes constituent 18,6\% (283 cas) des condamnations pour usurpation de la propriété de l'État commise avec la complicité d'une autre personne, offense impliquant des peines de 1 à 25 ans d'emprisonnement.

Comme mentionné plus haut, les femmes représentent 58,4\% (2 414 cas) des condamnés pour crimes économiques. De tels délits n'ont pas leur équivalent exact dans les pays capitalistes où la plupart des activités économiques de ce genre sont légales ou n'existent pas (Los, 1980, 1982 ; Grossman, 1977 ; Pomorski, 1977, 1978). Depuis que le Plan central régissant l'économie dans les pays communistes a force de loi, toutes les déviations économiques au Plan sont considérées comme crimes. Ceci implique naturellement une intervention légale massive de l'État dans l'économie, ainsi que le recours au droit pénal comme moyen essentiel de stimuler l'efficacité de la production économique.

La plupart des condamnations pour crime économique tombent dans la catégorie de la "spéculation » : l'accumulation de biens dans l'intention de les revendre à profit, un crime puni d'au plus trois ans de détention. En Pologne, 60\% (1 824 cas) des condamnations pour spéculations sans gravité étaient attribuables à des femmes. Pour comprendre la nature de cette sorte de crime, on doit se rappeler qu'en Pologne comme en Union Soviétique, il y a une pénurie chronique de nourriture et de produits de consommation. Le marché noir florissant et d'autres formes de redistribution illégale des biens sont donc la conséquence inévitable d'une économie centralisée et trop rigidement planifiée par l'État. Les produits à revendre peuvent être obtenus dans les magasins d'État, " sous la table ", des touristes étrangers et des marins, des fermiers indépendants ou directement des industries de l'État (dans ces derniers cas, d'autres offenses sont aussi commises).

Comme le journaliste britannique Stewart Steven l'indique dans son livre récent, The Poles «le marché noir est un des secteurs de l'économie polonaise qui fonctionne efficacement, qui témoigne de 
profits records et où il y a une croissance authentique » (Steven, 1982, $50)$.

Un autre type de crime économique qui figure dans les statistiques officielles concerne le rendement inhabituel d'un employé au sein d'une unité de l'économie étatique, rendement qui engendrerait la destruction, le mauvais emploi ou des dommages causés à l'économie socialiste. La peine, pour ce genre de crime, dépend du type particulier de délit et s'échelonne entre six mois et huit ans de prison. Le pourcentage de femmes trouvées coupables de ces crimes est de 56,9 en 1977 soit 574 cas. De tels crimes ne peuvent vraisemblablement être commis qui par des personnes détenant des postes de responsabilité ou appartenant à l'équipe de direction de leur unité économique. Tous ceux qui sont familiers avec l'organisation de l'économie polonaise ou soviétique, savent que la plupart des troubles et des pertes de production sont dus à une planification centrale défectueuse, un manque de synchronisation dans le fonctionnement des diverses unités économiques aussi bien qu'à l'existence d'un système inadéquat quant aux motivations économiques et aux critères de promotion (Besançon, 1981). Blâmer les cadres inférieurs pour une telle situation fait partie du processus politique de routine qui favorise les boucs émissaires et les purges rituelles du système.

Une autre catégorie de crimes que nous avons déjà mentionnés et pour lesquels les femmes sont susceptibles d'être poursuivies est le délit fiscal. Parmi ces crimes, l'introduction de la monnaie étrangère est de loin la plus fréquemment punie. En 1977, les femmes étaient l'objet de 31,4\% (484 cas) de ces condamnations alors qu'elles ne se voyaient réserver que $24,9 \%$ (52 cas) des condamnations pour infraction aux lois des douanes. Soulignons que les devises fortes exercent une attraction particulière, presque magique, sur les pays communistes surtout depuis que les citoyens ne peuvent plus officiellement acheter de devises occidentales. Et ce, en dépit du fait que plusieurs biens de consommation) aliments, cosmétiques, vêtements, autos, charbon, matériaux de construction, logements, etc.) rares sur le marché ouvert, sont immédiatement disponibles dans les «magasins pour devises étrangères » gérés par l'État. Ceci a eu comme résultat de développer dans le pays un système à double monnaie. Pendant que l'État cherche désespérément les devises étrangères pour importer des biens et régler 
sa dette extérieure, les citoyens, de leur côté, sont en quête désespérée des produits nécessaires. En conséquence, toute une foule complexe d'intermédiaires illégaux, hommes ou femmes s'occupant de trafic de devises et de marché noir, a surgi pour combler ces besoins insatisfaits et faciliter la circulation de l'argent.

Les monnaies étrangères sont introduites par des touristes, des contrebandiers, des fonctionnaires commerciaux étrangers corrompus et plusieurs autres marchés et échanges illégaux. La plus grande partie de cet argent aboutit dans les coffres de l'État via le réseau des magasins spéciaux, ce qui laisse croire que l'application de la loi dans ce domaine peut être légèrement sélective et manipulatrice. Il est également admis que les plus prospères marchands de devises opèrent avec la bénédiction des autorités. On croit qu'ils achètent leur immunité en payant des commissions spéciales.

Cependant, un autre type de crime où les femmes sont surreprésentées est la vente illégale d'alcool, crime pour lequel, selon les statistiques de 1977, 58,7\% (141 cas) des condamnées sont des femmes. L'alcoolisme compte parmi les problèmes sociaux les plus graves en Pologne et la demande pour l'alcool est toujours énorme. Cette demande est facile à satisfaire durant la journée alors que toutes les épiceries offrent un vaste assortiment de vodka, de bières et de vins à bon marché en dépit du rationnement de presque tous les autres produits comestibles. C'était du moins le cas en 1970. À partir des années 80, commence le rationnement de l'alcool qui a entraîné l'accroissement énorme des débits et du commerce clandestin ainsi qu'une ruée vers les magasins d'État où le rationnement ne s'applique pas. Le soir et le samedi, cependant, l'achat des spiritueux devient plus difficile. Les clients déjà intoxiqués ou les mineurs à qui l'État défend qu'on vende de l'alcool ont aussi des problèmes pour s'approvisionner. La demande d'alcool est suffisante, dans ces derniers cas pour justifier l'existence de vastes réseaux de vendeurs clandestins dont les approvisionnements viennent en partie de la fabrication illégale (qui était beaucoup plus faible en 77 qu'aujourd'hui), en partie des magasins ordinaires et en partie des magasins d'État où l'alcool est vendue à des prix relativement compétitifs même en calculant l'achat des monnaies étrangères sur le marché noir. Inutile de dire que l'économie de l'État profite de ces achats d'alcool par les trafiquants puisque inévitablement, ils drai- 
nent une grande quantité de devises étrangères du marché noir dans les goussets de l'État. En fait, il y a une nette corrélation entre les besoins de l'État en devises étrangères et les dimensions des approvisionnements en alcool des magasins qui n'acceptent pas la monnaie polonaise. Au cours de la période d'expansion économique et de modernisation de l'industrie polonaise qui reposaient lourdement sur l'importation de l'Occident, ces ventes se sont multipliées par plus de 24. Au cours de la même période, la production de vodka augmenta de $72,4 \%$. Le taux de consommation d'alcool s'est accru, au cours de ces six années, à un rythme plus élevé qu'en aucun pays européen et d'Amérique du Nord. Le nombre de personnes arrêtées pour inconduite due à l'état d'ébriété a presque doublé (Wald et al., 1981).

Ces données indiquent assez clairement que l'application de la loi est plutôt ambivalente vis-à-vis des ventes d'alcool. Il est intéressant de noter par ailleurs que la fabrication clandestine d'alcool va nettement à l'encontre des intérêts de l'État et de son monopole officiel sur toute production d'alcool. On croit que les marchands de spiritueux distillés illégalement sont poursuivis avec plus de diligence que ceux qui se contentent de servir d'intermédiaire entre la clientèle et le marché officiel.

\section{L'ÉMANCIPATION DE LA FEMME}

$\underline{\text { Retour à la table des matières }}$

Il y a tellement d'interprétations possibles de l'émancipation qu'il ne peut y avoir de réponses catégoriques quant à savoir si la femme polonaise est émancipée ou non. Une journaliste polonaise reste sceptique au sujet des avantages que la polonaise retire de sa glorieuse émancipation.

Jadis, les fonctions de l'épouse étaient clairement établies, fondées sur la tradition : l'homme gagnait le pain, elle s'occupait de tenir la maison. De nos jours, elle gagne de l'argent à ses côtés et continue à s'occuper de la maison comme ménagère bénévole. Des enquêtes auprès de la population polonaise démontrent que dans $83 \%$ des couples 
mariés, c'est la femme qui fait les courses et prépare les repas. Dans $78 \%$ des couples, la femme lave la vaisselle et dans $71 \%$ des couples, elle cire le plancher (Starczak-Kozkowska, 1982, 4, traduction libre).

Cependant, hors de la maison, l'implication de la femme au travail n'est pas de beaucoup inférieure à celle de l'homme, et les femmes constituent 46\% de la force de travail. Entre 25 et 40 ans, de 75 à 80\% des femmes qui ont un enfant détiennent un emploi régulier à l'extérieur du foyer alors que c'est le cas de $58 \%$ des mères de 4 enfants (Plenska, 1980, 465).

Sans doute, la vie est-elle très difficile pour les mères polonaises au travail. Les activistes occidentales des mouvements de libération des femmes leur font un tort considérable en diffusant de l'information non fondée au sujet des excellents soins gratuits aux enfants et des services d'assistance aux familles qui existent dans les pays communistes. Rien n'est plus éloigné de la vérité et des luttes quotidiennes des mères polonaises.

Par exemple, les mères au travail qui parviennent à résoudre le problème de garde des enfants pendant leur absence du foyer doivent encore attendre en file chaque jour à l'épicerie en plus d'assumer les tâches requises pour le fonctionnement normal d'un ménage dont l'approvisionnement et les conditions économiques sont problématiques. Une recherche indique que les mères de deux enfants passent sept heures par jour à travailler à la maison en plus de leurs huit heures de travail à l'extérieur, et ce, six jours par semaine. Cet horaire, notons-le, ne comprend pas les heures passées à faire les courses (StarczakKozlowska, 1982, 4).

À cause des besoins d'une économie qui s'est développée rapidement dans la période d'après-guerre, la femme polonaise a souvent dû remplir deux postes à plein temps au lieu d'un seul qu'elle occupait traditionnellement. Son appartenance à la force du travail est de plus dictée par la volonté des nouvelles autorités communistes de la soumettre, comme principal agent de socialisation, à un endoctrinement idéologique, qui peut plus facilement être mené en milieu de travail qu'au sein du foyer. 
Il existe cependant une autre facette à la libération sociale de la femme qu'on ne peut décrire en termes si tranchés. Par exemple, plusieurs femmes apprécient leur travail et profitent pleinement des avantages qu'il leur donne. De plus, les Polonaises sont aussi instruites sinon plus que les hommes, ce qui démontre jusqu'où la société est allée dans l'idée d'égalité des chances de formation pour les deux sexes. Cependant, il demeure une très forte distinction entre les métiers masculins et féminins. On trouve peu de garçons dans les écoles professionnelles de coiffure, de vente, de secrétariat, de nursing, etc. De même, très peu de filles suivent des cours de mécaniciens ou de techniciens.

Au niveau universitaire, les cours de langue, de pédagogie et de bibliothéconomie, de même que les études de médecine (qui, pour longues qu'elles soient, ne conduisent qu'à des salaires relativement peu élevés) comptent un nombre prédominant de femmes. Les hommes continuent d'être majoritaires dans les écoles d'ingénieurs qui mènent aux postes les mieux rémunérés.

On sait qu'en dépit d'une instruction égale et, parmi les plus jeunes, même supérieure, les femmes gagnent environ $70 \%$ du salaire des hommes (Coser, 1980), elles occupent des emplois, sans possibilité d'avancement, et sont très rarement promues à des postes de commande. De plus, elle sont pour ainsi dire absentes des organismes exécutifs du parti communiste et du gouvernement et demeurent nettement sous -représentées au Parlement (20\% des députés).

Bien que les femmes aient accepté l'importance du travail comme un indice de leur statut, elles n'en demeurent pas moins conscientes qu'elles sont aussi évaluées sur la façon dont elles remplissent leur rôle de mère. Il en découle qu'elles sont constamment soumises aux tensions dues à un sérieux conflit de rôle, à une personnalité déviée et à une conscience troublée, quel que soit leur mode d'adaptation à ces pressions contradictoires. Cela signifie aussi qu'elles subissent encore l'influence des modèles culturels traditionnels, des attentes et des contrôles sociaux qui mettent de l'avant des normes et des valeurs différentes pour les hommes et pour les femmes. Essentiellement, leur responsabilité première vis-à-vis de l'éducation et la formation morale de leurs enfants renforce leurs liens avec l'Église et les valeurs tradi- 
tionnelles de la communauté qui sont généralement perçues comme menacées par la culture politique opportuniste et hypocrite imposée aux enfants à travers l'école et les médias.

\section{L'ÉMANCIPATION DE LA FEMME ET LE CRIME : LES ANNÉES D'APRÈS-GUERRE}

$\underline{\text { Retour à la table des matières }}$

De 1940 à 1960, les statistiques révèlent que l'augmentation de la délinquance des femmes par rapport à celle des hommes ne peut pas s'expliquer simplement en termes d'émancipation féminine. L'émancipation, au sens propre, implique un processus en soi, un combat pour une plus grande participation et une libération. Cependant, l'accroissement rapide du travail des femmes et leur participation aux rôles traditionnellement masculins en Pologne ont pour cause divers facteurs extérieurs et objectifs. Parmi les circonstances qu'on oublie souvent, notons la plus grande proportion d'hommes que de femmes parmi les six millions de Polonais qui ont été tués durant la guerre. De plus, un plus grand nombre d'hommes que de femmes étaient à l'extérieur du pays à la fin de la guerre, lors de la prise de pouvoir communiste, à cause de leur engagement avec les forces armées polonaises dans le combat contre l'armée allemande ailleurs en Europe. Alors que de nombreux vétérans de guerre s'établissaient à l'Ouest, des milliers de ceux qui retournaient ou qui étaient membres de l'Armia Krajowa ${ }^{3}$ furent l'objet de vigoureuses persécutions et, souvent, de longs emprisonnements.

Le nombre de morts parmi les hommes au cours de la guerre et de l'après-guerre explique, jusqu'à un certain point, la nécessité pour les femmes de s'impliquer dans la réorganisation et l'économie du pays.

3 L'Armée de l'intérieur des forces clandestines. 
Ainsi, cela met en évidence l'aspect purement démographique de la plus forte proportion de femmes qui figurent aux statistiques criminelles officielles. De toutes façons, on doit se rappeler que l'augmentation de l'activité professionnelle des femmes ne fut qu'un facteur parmi plusieurs transformations traumatisantes d'ordre social et économique qu'a vécues la Pologne d'après-guerre. L'industrialisation intense et la collectivisation de l'agriculture calquées sur le modèle stalinien, supposaient une complète subordination des besoins et des valeurs individuelles aux exigences de l'économie et de l'idéologie.

L'exode massif de la population rurale vers les villes et les nouveaux centres industriels ont amené la destruction des liens communautaires et des contrôles traditionnels. Des jeunes gens et des jeunes femmes quittèrent leurs villages par milliers pour chercher ailleurs des vies meilleures et de nouveaux débouchés économiques ${ }^{4}$. Alors que la plupart des immigrants non qualifiés trouvaient du travail dans la construction des nouveaux complexes industriels, plusieurs femmes, incapables de trouver dans les services encore mal développés et refusant les tâches harassantes de la construction finissaient par se tourner vers la prostitution. De cette façon, elles répondaient aux besoins des grandes communautés d'hommes, éloignés à la fois de leurs familles et de leurs coutumes rurales et de la culture et des facilités urbaines.

Les modifications de frontières de l'après-guerre en Pologne ont provoqué encore un autre déplacement d'importance de la population, principalement des parties orientales du pays dont l'Union Soviétique s'était emparée vers les territoires occidentaux reconquis. Les nouveaux occupants, totalement déracinés et aliénés culturellement, furent confrontés aux dures réalités d'une terre dévastée par le retrait des troupes allemandes, dépourvus de toute infrastructure sociale et aux prises avec l'hostilité des divers groupes ethniques et culturels jetés les uns contre les autres par les forces cruelles de l'histoire ${ }^{5}$. Pour plu-

4 Entre 1946 et 1975, 3,5 millions de personnes passèrent des régions rurales à la ville (Dyoniziak et al., 1978 : 57)

5 Selon les statistiques, 4,5 millions de personnes se sont établies dans les régions de l'ouest autour de 1950. Presque trois millions venaient des autres parties de la Pologne, alors qu'un million et demi étaient rapatriés de l'Union Soviétique (Dyoniziak, 1978 : 55). 
sieurs raisons, l'émoi causé par leur déplacement forcé fut si grand qu'ils devinrent des nomades typiques, instables, incapables de s'établir nulle part ni de se recréer des racines dans des terres nouvelles. Comme les documents polonais sur le sujet l'attestent, tous les indicateurs de la désorganisation sociale comme l'alcoolisme, l'hospitalisation psychiatrique, le crime, la délinquance juvénile et les familles brisées atteignent des records dans ces régions (Maroszek, 1975 ; Kobus, 1975 ; Krycka, 1978). En termes de statistiques, l'accroissement incroyable du crime dans cette partie de la Pologne a eu un impact significatif sur le taux national de la criminalité et de la délinquance au cours de cette période. Cela a sans doute affecté considérablement l'image générale de la délinquance des femmes tout particulièrement à mesure que les contrôles habituels et les valeurs traditionnelles perdaient de leur importance sous l'impact des conflits culturels et de l'anomie.

En somme, il semblerait plutôt inadéquat de relier les augmentations proportionnelles temporaires de la criminalité des femmes de l'après-guerre qu'elles soient vraies ou fausses, à l'émancipation sociale de la femme sans considérer les circonstances très complexes et les transformations sociales de cette période agitée de l'histoire de la Pologne.

\section{L'ÉMANCIPATION DE LA FEMME ET LE CRIME : LES VINGT DERNIÈRES ANNÉES}

$\underline{\text { Retour à la table des matières }}$

Comme nous l'avons dit précédemment, le rapport général des condamnations des femmes et des hommes est assez faible (à peu près 1 :9). Cependant, ce rapport, en ce qui concerne les crimes économiques et les délits non violents contre la propriété de l'État est considérablement plus élevé. En 1977, par exemple, 6073 condamnations de femmes (sur un total de 14 059) concernent des crimes non violents 
contre la propriété de l'État ou des actes contre l'économie de l'État ${ }^{6}$. Cela représente $33,6 \%$ de toutes les condamnations pour ces crimes, ramenant la proportion des femmes par rapport aux hommes pour ces crimes à $1: 2$. Le reste des condamnations qui comprennent les crimes contre la personne, la liberté, la famille, la propriété privée, le fonctionnement de l'État et des institutions sociales constituent $6,7 \% \mathrm{du}$ nombre total des condamnations pour ces crimes ; le rapport femmehomme qui en découle est de $1: 14$. L'analyse qui suit tentera d'interpréter et d'expliquer l'écart manifeste qui existe entre les taux rapportés de délinquance des femmes dans les domaines plus traditionnels de la criminalité et ceux qui touchent aux secteurs plus directement reliés aux caractéristiques propres à l'organisation de l'économie communiste.

Il découle logiquement de la position sociale de la femme et de la nature de son émancipation qu'elle continue à être beaucoup plus soumise aux contrôles traditionnels que l'homme. Son rôle de mère lui impose la tâche extrêmement difficile de préserver et de transmettre d'une génération à l'autre les valeurs menacées de la culture polonaise qui assure la survivance de ses traditions repose très fortement sur la préservation du modèle traditionnel de la mère polonaise. De plus, l'expérience historique de la femme polonaise qui, si souvent par le passé, a su allier avec succès ses fonctions maternelles à ses devoirs civiques permettant ainsi à la Pologne de traverser de longues périodes de guerre, de division et d'occupation, semble soutenir et faciliter ses efforts actuels. Tout en exigeant des femmes héroïsme et persévérance, ces fonctions équilibrent en quelque sorte leur vie ; les échecs sur un plan peuvent être compensés par les succès sur un autre et les gratifications découlant tantôt de l'une, tantôt de l'autre activité peuvent leur fournir la force nécessaire pour poursuivre leur impossible tâche.

L'importance du statut des hommes, par ailleurs, semble diminuer à mesure que le travail des femmes rend moins nécessaire leur rôle de

6 Ceci comprend l'usage illégal des biens de l'État, l'usurpation d'une propriété de l'État de grande valeur ou avec l'aide de complices, le recel des biens volés à l'État, l'accomplissement inadéquat de ses tâches nuisant à l'économie de l'État, la spéculation, les délits fiscaux et la vente illégale d'alcool. 
pourvoyeur financier de la famille et leur engagement dans l'éducation des enfants porte toujours une marque de féminité, une atteinte à leur ego masculin, et ce, dans plusieurs couches sociales. En somme, alors que le sentiment de responsabilité économique et familiale de la femme s'accroît, le sens de responsabilité et d'indispensabilité pour l'homme semble s'atrophier.

Le manque d'occasions de s'impliquer socialement et politiquement à la base (sauf pendant la période du mouvement Solidarité) et le haut niveau d'aliénation politique et économique dans un pays gouverné par un État totalitaire et centralisateur contribue à l'insécurité et à la relative futilité de leur position sociale. Le taux extrêmement élevé d'alcoolisme, les niveaux relativement hauts de crimes violents, les taux alarmants de crimes contre la famille et la jeunesse y compris les sévices envers les enfants et les épouses et le non-paiement des pensions alimentaires, tendent à établir que l'homme voit vraiment et de façon notoire, diminuer son sens des responsabilités, son importance sociale et sa sécurité psychologique.

Le taux de suicide aussi semble fournir une indication supplémentaire du remarquable niveau de la persévérance féminine, de son altruisme et de sa préoccupation du bien-être des autres qui contrastent avec les sentiments masculins de marginalité relative et de perte de statut. Bien que les taux de suicides des hommes soient systématiquement plus élevés dans presque tous les pays, l'écart entre les taux de suicides des hommes et des femmes est exceptionnellement grand en Pologne où les hommes s'enlèvent la vie cinq fois plus souvent que les femmes ${ }^{7}$. Dans la plupart des pays qui publient des statistiques sur le suicide, cette proportion varie entre $1: 2$ à $1: 3$.

Une compréhension de ces différences de statut conditionnées par la culture entre la femme et l'homme polonais aide à expliquer et à interpréter la faible augmentation de la participation féminine à la criminalité conventionnelle à comparer aux taux de criminalité des hommes. Il n'existe cependant pas de recherches qui permettent d'éva-

7 Par exemple, à taux de suicide égal en Pologne et au Canada, les femmes canadiennes sont deux fois plus susceptibles de s'enlever la vie que les femmes polonaises (Sarosz, 1980 ; Sarosz, 1978). 
luer avec exactitude les statistiques officielles à cet égard. Bien qu'il n'y ait aucune raison de supposer que la police polonaise soit moins paternaliste envers les femmes que ses collègues des pays occidentaux, il est impossible de conclure que cette attitude provoque des partis pris systématiques dans l'application de la loi. Étant donné le très haut pourcentage de femmes juges et procureurs généraux, ces influences peuvent être quelque peu minimisées au niveau des tribunaux.

Ces raisons n'expliquent cependant pas le taux relativement élevé des condamnations pour des délits économiques non conventionnels. Selon les statistiques des tribunaux de 1977, seulement $10 \%$ de tous les hommes condamnés, mais plus de $40 \%$ des femmes condamnées furent trouvés coupables des délits énumérés plus haut (Note 6).

Bien qu'il puisse exister un parti pris systématique contre les femmes dans l'application de la loi pour ce genre de crime, il y a aussi d'autres explications plausibles des raisons pour lesquelles ces différences peuvent refléter, au moins partiellement, certains processus sociaux et culturels bien réels. Les transformations radicales dans l'organisation économique et la question de la propriété en Pologne, accompagnées de la criminalisation de plusieurs nouveaux secteurs reliés à l'activité économique, n'ont jamais été mises en parallèle avec les changements correspondants dans la conscience sociale ci la culture normative officieuse de la société.

L'absence d'efficacité économique et de légitimité politique aussi bien que l'idéologie ouvertement athée de l'ordre nouveau ont empêché la fusion naturelle des anciennes et des nouvelles valeurs. Il semble assez probable que, tandis que les femmes continuent à suivre les règles traditionnelles et les normes qui régissent les crimes plus conventionnels, elles ne se sentent pas liées dans les nouveaux secteurs où ne s'appliquent ni les normes ni l'autorité de la tradition. De plus, ces nouveaux secteurs sont étrangers au milieu familial et ne semblent pas avoir de lien avec la réalisation de la femme dans soit rôle culturel de mère. Ce dernier est soumis à la morale cohérente ci familière des rapports interpersonnels, destinée à protéger la santé et la vie humaine, la famille et la propriété privée. Ce fossé entre la nouvelle et l'ancienne morale se retrouve aussi bien chez les hommes que 
chez les femmes, mais les premiers semblent moins attachés à la morale traditionnelle à cause de leur socialisation différente et de leur moins grande implication dans l'éducation morale des enfants.

Les crimes économiques aussi bien que les crimes ordinaires contre la propriété de l'État sont très répandus partout en Pologne comme d'ailleurs dans tous les autres pays communistes. La plupart d'entre eux ne donnent lieu à aucune poursuite, soit parce qu'ils prennent des proportions incontrôlables et endémiques ou à cause de l'importance politique de leurs auteurs et donc de leur impunité. Comme nous l'avons déjà dit, l'application de la loi dans le secteur du crime économique est très sélective et manipulatrice. De même, elle est sujette à beaucoup plus d'interférence politique, spécialement de la part des élites locales du parti, que l'application de la loi dans le secteur de la criminalité conventionnelle.

La véritable signification des statistiques judiciaires concernant le crime économique est très difficile à interpréter. Ce qui est intrigant, par exemple, c'est la grande proportion de femmes (56,9\%, en 1977) condamnées pour insuffisance de rendement au travail dans une unité économique de l'État, ce qui nuit considérablement à l'économie. Les femmes ont aussi un taux relativement élevé de condamnations pour usage illégal des biens de l'État qu'elles doivent administrer (32,3\%) et pour vol des biens publics de valeur (20,2\%). Il est évident que les gens qui occupent des postes plus élevés, des postes de direction, ont davantage d'occasions de causer du tort à l'économie que les employés subalternes. Puisque, de toute manière, les femmes ne se voient que rarement attribuer ces hauts postes, il se peut qu'elles soient souvent accusées à titre de boucs émissaires commodes. De même, dans les cas de complicité, la loi ne distingue pas entre les responsabilités des individus impliqués et très souvent, on choisit de poursuivre les participants secondaires qui doivent alors assumer la pleine responsabilité de crimes commis par des bandes comprenant souvent des fonctionnaires relativement haut placés (Podemski, 1981). Comme les femmes travaillent surtout comme caissières ou comptables, elles sont plus susceptibles d'être au fait et par conséquent de participer aux opérations de tels groupes criminels, mais leur rôle véritable peut être assez limité. Néanmoins, elles constituent des cibles assez faciles pour les soupçons et les poursuites sans conséquences politiques qui épargnent 
généralement les complices vraiment influents et puissants, On ne doit cependant pas conclure que les femmes ne peuvent pas jouer de rôle important dans de telles organisations criminelles, mais plutôt que, sur le plan statistique, elles sont rarement en position de tirer de réels profits de leur participation à de tels crimes économiques.

Pour les délits mineurs de transaction qui sont, selon les données officielles, les délits typiquement féminins (les femmes comptent 60\% de condamnations en 1977), on doit remarquer que plusieurs sortes de transactions peuvent être effectuées par des femmes, et ce, de plusieurs façons. La sorte la plus commune et visible est J'achat de produits rares dans les magasins d'État, chez les paysans ou les employés d'entreprises de l'État et la revente à plus hauts prix aux coins des rues, dans des marchés privés ou à travers d'autres canaux du marché noir. Cela ne requiert pas beaucoup d'organisation, ni aucune habileté spéciale et c'est facilement repérable puisque le délinquant participe directement à toutes les transactions (achat illégal, vente illégale, etc.) Ce genre de spéculation compte numériquement beaucoup plus d'adeptes féminins.

Les femmes sont très familières avec les opérations du marché, les produits manquants et la mentalité des acheteurs parce qu'elles approvisionnent elles-mêmes leur famille et que les frustrations dues aux constantes pénuries font partie de leur vie quotidienne. De plus, les femmes qui consacrent tout leur temps à ce commerce illégal ont le net avantage sur les hommes de ne pas attirer l'attention ni de subir de harcèlement de la part de la police parce qu'elles n'ont pas d'emploi régulier ${ }^{8}$.

En plus de ces petits commerçants de biens usuels de consommation, il existe aussi un réseau beaucoup mieux organisé de vendeurs en gros qui trafiquent sur une haute échelle, les biens volés à l'État (par exemple avec des hommes d'affaires privés), les oeuvres d'art (habituellement passées outre frontière en contrebande), les appartements,

8 Il vaut la peine de mentionner qu'une nouvelle toi antiparasite adoptée en 1982 en Pologne, rend criminel, pour un homme, le fait de demeurer de façon persistante sans emploi socialement utile. Cette loi ne s'applique pas aux femmes. 
les automobiles, la machinerie agricole, les matériaux de construction ou tout autre produit dont le marché est contrôlé par des règlements politiques. Pour protéger la mise en vente sur le marché noir de grandes quantités de ces produits, de tels réseaux doivent s'affilier des fonctionnaires haut placés à l'échelon local et même central, puisque leur coopération est essentielle au succès de leurs opérations et à leur impunité relative. À cause de l'inefficacité de l'application de la loi et à cause de la protection politique accordée à ce genre de spéculation, il est difficile de déterminer la participation des femmes et leur rôle. Il n'y a pas de doute cependant que ce genre de commerce est beaucoup plus nuisible à l'économie et profitable à ses auteurs que les trafics de chaussettes au coin des rues, attribués aux femmes et qui sont hautement criminalisés.

Enfin, comme nous le mentionnions plus haut, les femmes sont souvent poursuivies pour commerce de monnaies étrangères (31,4\%) et ou vente illégale d'alcool (58,7\%). L'État, disions-nous, a tout intérêt à promouvoir ces crimes aussi longtemps qu'il peut garder le contrôle des profits et assurer la canalisation efficace du plus gros des devises fortes impliquées vers ses propres coffres. Cela requiert habituellement à un certain degré la coopération des trafiquants avec la police ou les agents secrets. De plus, puisque les deux crimes sont relativement évidents pour la société, certains délinquants doivent être désignés à la poursuite pour détourner tout soupçon du public quant à la corruption totale de la police ou sa complète inertie.

Les femmes qui s'occupent des devises fortes sont souvent des prostituées travaillant dans des hôtels de luxe et des restaurants qui attirent beaucoup de visiteurs étrangers. Non seulement demandentelles à être payées de leurs services en devises fortes, mais elles sont aussi utilisées par leur souteneur, eux-mêmes commerçants de monnaie, pour faire des échanges illégaux pour leurs clients. D'autre part, les femmes engagées dans le trafic et la fabrication de l'alcool sont souvent d'anciennes prostituées que l'âge ou l'état de santé empêchent de travailler, mais qui n'ont aucune situation légitime dans la société. Elles peuvent ordinairement compter sur leurs anciens clients et amis pour répandre l'information au sujet de leur nouveau commerce et pour s'assurer un nombre suffisant d'acheteurs. La condamnation des prostituées ou des ex-prostituées arrête leurs activités criminelles pour 
la durée de leur emprisonnement, mais elles reprennent leur commerce d'ordinaire sitôt qu'elles sont libres, n'ayant aucune protection légale, aucun groupe de référence ni de véritable choix économique viable. Ainsi, leur incarcération sporadique ne dérange pas l'équilibre du réseau adroitement organisé de services et profits mutuels dans un système hautement intégré de marchés licites et illicites.

En somme, les statistiques officielles qui l'ont état de la participation relativement élevée de la femme aux délits économiques non conventionnels doivent être examinées avec circonspection. Bien qu'il ne fasse aucun doute que les femmes participent assez activement à diverses sortes de délits économiques et fiscaux, il faut demeurer conscient que c'est un domaine où l'application de la loi est fortement sélective et influencée par la politique.

\section{L'ÉMANCIPATION DE LA FEMME : DISCUSSION}

$\underline{\text { Retour à la table des matières }}$

Les femmes en Pologne sont instruites, travaillent à la fois à la maison et à l'extérieur et sont respectées par les hommes. Les enquêtes d'opinion publique démontrent que les femmes sont d'orientation plus altruiste et accordent plus de valeur au bonheur de la vie familiale que les hommes (Reszke, 1978). De plus, les attentes des parents sur le plan académique sont plus fortes pour leurs filles que pour leurs fils. Le choix d'une carrière pour les filles et pour leurs parents est cependant réaliste et adapté aux exigences de l'éducation des enfants et des devoirs ménagers (c'est-à-dire un travail qui n'est pas trop épuisant physiquement, qui exclut les déplacements, les horaires mobiles, les grandes responsabilités, etc. (Reszke, 1978).

Les femmes semblent constituer une solide couche sociale moyenne à l'intérieur de la structure professionnelle polonaise. Elles travaillent comme enseignantes ou appartiennent au personnel médical, la plupart d'entre elles occupent des positions intermédiaires dans 
les services, les institutions financières et, surtout, dans l'administration. Leur présence aux échelons inférieurs et supérieurs de la hiérarchie est plus rare. Elles semblent être plus résistantes et moins vulnérables à la frustration qu'engendre la structure formelle de la société. En dépit de toutes les pressions et des difficultés encourues pour mener à bien leur double rôle, les femmes réussissent probablement à surmonter les échecs professionnels et la déception à cause des gratifications qu'apporte l'éducation des enfants et vice-versa,

Les hommes ne paraissent pas avoir un tel choix. Leur responsabilité envers leur famille comme pourvoyeurs financiers a remarquablement diminué, alors que leur rôle dans l'éducation des enfants demeure marginal. Leurs insatisfactions sur les plans de la formation et du travail n'ont pas de compensation et ils demeurent donc plus susceptibles de se mêler à la sous-culture criminelle ou de mener une existence marginale d'alcooliques, d'instables, de vagabonds. Par ailleurs, les hommes dont les aspirations et l'initiative ne peuvent se réaliser dans une économie communiste sont plus enclins à élaborer des complots en vue d'introduire des objectifs capitalistes, de donner une motivation de profit à d'audacieuses entreprises économiques. Contrairement aux femmes, leur dynamisme dans l'obtention de leur satisfaction personnelle, leur statut et le succès économique ne sont pas effectivement limités par les menues tâches ménagères et la responsabilité du développement moral de leurs enfants.

Durant les années des activités légales du syndicat libre Solidarité de 1980-1981, les Polonais furent témoins de la découverte massive des abus de pouvoir des fonctionnaires communautaires à tous les niveaux de la hiérarchie économique et politique. La liste des noms des fonctionnaires poursuivis ou dénoncés pour corruption et crime économique grave confirme une fois de plus l'absence des femmes parmi les échelons supérieurs de la criminalité. Ironiquement, il y avait aussi peu de femmes parmi les prisonniers politiques arrêtés et détenus après l'imposition des mesures de guerre en Pologne. De même, les femmes étaient remarquablement sous-représentées parmi ceux qui étaient dénoncés et condamnés pour des crimes politiques en vertu de la loi martiale. Cela semble concorder avec la distribution selon le sexe parmi les officiels de Solidarité aussi bien que les perceptions 
officielles du degré de danger posé par les hommes et les femmes contestataires ${ }^{9}$.

En conclusion, il semble clair que les femmes constituent une mince minorité à l'intérieur du leadership du mouvement démocratique, comme d'ailleurs au sein de l'appareil du parti communiste, de l'élite économique dirigeante, de l'armée et, par définition, dans la hiérarchie de l'église catholique. Non seulement les sommets de pouvoir mais aussi ses abus semblent inaccessibles aux femmes polonaises. Il n'appartient pas au chercheur de juger qui est gagnant ou perdant dans le jeu de la ségrégation des sexes et des stéréotypes sexuels au royaume du pouvoir.

\section{BIBLIOGRAPHIE}

$\underline{\text { Retour à la table des matières }}$

ADLER, F. (1977). " The Interaction between Women's Emancipation and Female Criminality », International Journal of Criminology and Penology, vol. 5, no 2, pp. 101-112.

BERTRAND, Marie-Andrée (1979). La femme et le crime. Montréal : L'Aurore. [Texte disponible dans Les Classiques des sciences sociales. JMT.]

BESANÇON, A. (1981). Anatomie d'un spectre. L'économie politique du socialisme réel. France : Calmann-Lévy.

COSER, R.L. (1980). «Women and Work ». Dissent, vol. 27, no 1.

9 Les femmes constituent une petite minorité parmi les délégués au Congrès national de Solidarité en 1981 et sont élues à peu de postes. ce fait a contribué à une prise de conscience quant au statut inégal de la femme dans la société. 
DYONIZIAK, R., J. MIKULOWSKI-POMORSKI, and Z. PUCEK, (1978), Wspolczesne Spoleczenstwo Polskie (Contemporary Polish Society). Warszawa : PWN.

GROSSMAN, G. (1977). "The Second Economy of the U.S.S.R. ». Problems of Communism, September-October, vol. 26, no 5, pp. 25-40.

HAGAN, J., H. SIMPSON, and A.R. GILLS. « The Sexual Stratification of Social Control : A gender-Based Perspective on Crime and Delinquency ». British Journal of Sociology, Vol. 30, no I (March).

JAKUBOWSKA, A. (1981). «Fikcyjne rownouprawnienie »(Fictitious Equality). Sztandar Mlodych, April :8.

JAROSZ, M. (1978). "Le suicide en Pologne ». Revue d'études comparatives : Est-Ouest. vol. 9, no 4, pp. 65-101.

JAROSZ, M. (1980). "Zostawic i odejsc ? » (To Leave and Abandon ?). Kobieta i Zycie. no 34/1644, 24 November : 4.

KOBUS, A. (1975). »Nicktore zagadnienia przestepczosci nieletnich w PoIsce w latach 1959-1973 ». (Some problems of juvenile delinquency in Poland in 1958-1973), pp. 103-122, in M. Jarosz, ed., Wyhrane Zagadnienia Patologii Spolecznej (Selected Problems of Social Pathology). Warszawa : GUS.

KRYCZKA, P. (1978), " Some Phenomena of Social Pathology in Poland ». The Polish Sociological Bulletin, no 2, 4, pp. 101-109.

BOS, M. (1980). « Economic Crimes from a Comparative Perspective ", pp. 251-293, in G.R. Newman, ed., Crime and Deviance. A Comparative Perspective. Beverly Hills, California : Sage.

BOS, M. (1982). "Crime and the Economy in the Communist Countries ", pp. 121-137, in P. Wickman and T. Dailey, eds., WhileCollar and Economic Crime. Lexington, Massachussetts : Lexington Books. 
MANKIEWICZ, M. (1981a). "Duzo sie teraz robi dla dobra dziccka " (Much is done now for children), Kobieta i Zycie, no 36/1596, 6 September : 3, 23.

MANKIEWICZ, M. (1981 b). « Tajemnica cudownego rozmnozcnia po polsku » (The secret of miraculous multiplication), kobieta $i$ Zycie, no 40/1600, 4 October : 4-5.

MAROSZEK, B. (1975). « Delinquance juvénile et développement socio-économique en Pologne ", in I. Chirol, B. Maroszek et al., Délinquance juvénile et développement socio-économique. La Hague: Mouton.

PLENSKA, D. (1980). "La criminalité féminine en Pologne », Canudian Journal of Criminology, vol. 22, no 4, pp. 464-475.

PODEMSKI (1981). « Amnestia bez schematow (Amnesty without schemes), Polilyka, no 21, 23 May : 3.

POMORSKI, S. (1977). "Criminal Law Protection of Socialist Property in the U.S.S.R. », pp. 223-258, in D.B. Barry et al., eds., Soviet Law after Stalin. Part I : The Citizen and the State in Contemporary Soviet Law. Leyden : A.W. Sijthoff.

POMORSKI, S. (1978). "Crimes against the Central Planner: Ochkovtiratel'stvo' ». pp. 291-317, in O. D. Barry et al., eds., Soviet Law after Stalin. Part II : Social Engineering Through Law. Alpen aan den Rijn : §ijthoff \& Noordhoff.

RESZKE, I. (1978). « Kulturowe i biopsychiczne uwarunkowania feminizacji zawodow (Cultural and bio-psychological factors in feminization of occupation), Studia Socjologiczne, no 3 (78) : 151-181.

SIDORCZUK, B. (1981). "A gdzie sa kobiety » (Where are women ?), Kobieta i Zycie, no 24/1584, 14 June : 3, 15.

SMART, C. (1977a). Women, Crime and criminology. London : Routledge and Kegan Paul. 
SMART, C. (1977b). «Criminological Theory : Its Ideology and Implications Concerning Women ", British Journal of Sociology, vol. 28, no 1, march, pp. 89-100.

STARCZAK-KOZLOWSKA, K. (1982). «Zycie w galopie » (Life in a Gallop), Fakty, vol. 24, no 39/856, pp. 4-5.

STEVEN, S. (1982). The Poles. New York : Macmillan.

WALD, I. et al. (1981). Raport o Problemach Polityki w Zakresie Alkoholu (Report on Policy Concerning Alcohol). Warszawa: Rada Ministrow. Who's Who. What's What. Solidarnosc. Leksykon Zwiazkowy (1981). Gdan'sk : BIPS.

Fin 